\title{
Beyond Phasors: Modeling of Power System Signals Using the Hilbert Transform
}

\author{
Asja Derviškadić, Member, IEEE, Guglielmo Frigo, Member, IEEE, Mario Paolone, Senior Member, IEEE
}

\begin{abstract}
Modern power systems are at risk of largely reducing the inertia of generation assets and prone to experience extreme dynamics. The consequence is that, during electromechanical transients triggered by large contingencies, transmission of electrical power may take place in a wide spectrum well beyond the single fundamental component. Traditional modeling approaches rely on the phasor representation derived from the Fourier Transform (FT) of the signal under analysis. During large transients, though, FT-based analysis may fail to accurately identify the fundamental component parameters, in terms of amplitude, frequency and phase. Taking inspiration from the theory on analytic signals, this paper proposes a different approach to model signals of power systems electromechanical transients based on the Hilbert transform (HT). We compare FT- and HT-based approaches during representative operating conditions, i.e., amplitude modulations, frequency ramps and step changes, in synthetic and real-world datasets. We further validate the approaches using a contingency analysis on the IEEE 39-bus.
\end{abstract}

Index Terms-Hilbert Transform, Instantaneous power, Phasor analysis, Power system modeling, Transient analysis

\section{INTRODUCTION}

Power systems are rapidly evolving towards low-inertia networks and system operators are facing new challenges to operate their grids safely [1]-[5]. Specifically, they are facing a dramatic increase in renewable energy sources and inverterconnected devices that, as such, do not provide any inertia to filter dynamics originated by power system disturbances [6][9]. In the so-formed inertia-reduced power grids, phenomena that used to be exceptional in traditional networks, such as frequency modulations, rapid (i.e., sub-second) frequency variations or sudden amplitude steps, are more likely to be experienced, and have been identified and documented by system operators [10], [11].

A clear example happened on September 2016 when the South Australian system faced a severe blackout because a strong windstorm hit the region while half of its power consumption was fed by wind generation [10]. Fig. 1(a) shows the frequency as recorded by Phasor Measurement Units (PMUs) installed in that area. The frequency experienced a large drop of almost $4 \mathrm{~Hz}$ in about 0.7 seconds, with an estimated Rate-of-Change-of-Frequency (ROCOF) of roughly $-6.25 \mathrm{~Hz} / \mathrm{s}$. From a theoretical perspective, it is worth investigating whether these operating conditions can be thoroughly

The Authors are with the École Polytechnique Fédérale de Lausanne EPFL, CH-1015, Lausanne, Switzerland (e-mail: asja.derviskadic@epfl.ch).

This project is carried out within the frame of the Swiss Centre for Competence in Energy Research on the Future Swiss Electrical Infrastructure (SCCER-FURIES) with the financial support of the Swiss Innovation Agency (Innosuisse - SCCER program).
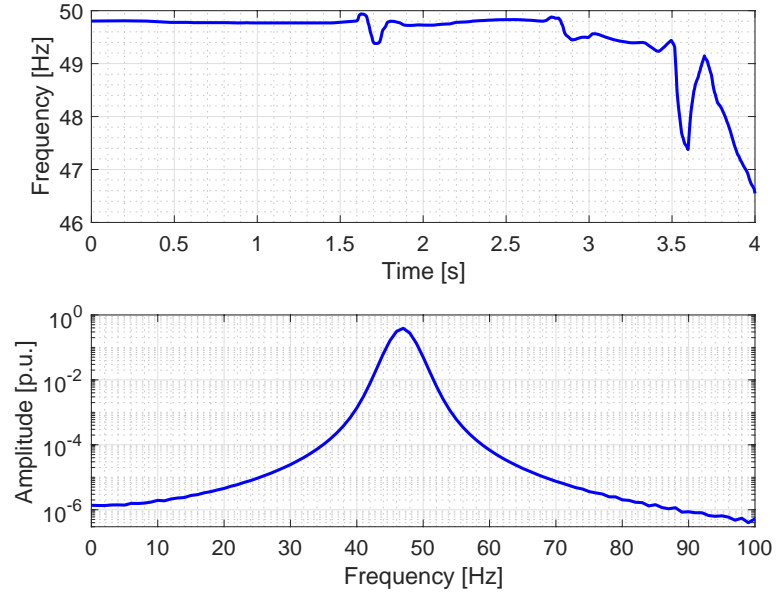

Fig. 1. Frequency estimated by PMUs during the Australian blackout on September 28, 2016 [10] (a) and normalized spectrum of a single-tone signal characterized by ROCOF of $-6.25 \mathrm{~Hz} / \mathrm{s}$ computed using the DFT (b).

described using traditional power system analysis tools that refer to the concept of phasor and assume signals to be in steady-state and characterized by narrow-banded spectra [12]. Typically, phasor parameters are determined using the Fourier Transform (FT): the frequency, amplitude and phase of the phasor associated to the fundamental component can be inferred from the FT coefficients laying around the nominal system frequency [13]. However, during dynamic events, the FT-based representation may not allow for unequivocally identifying the parameters of the fundamental component [14]. Same considerations hold when applying frequency tracking techniques, such as the Short-Time FT (STFT) [15], or when representing the signal as a series of dynamic coefficients via Taylor-Fourier expansions [16]. In this respect, Fig. 1(b) represents the spectrum of a single-tone signal at $50 \mathrm{~Hz}$, whose frequency is decaying with a ROCOF of $-6.25 \mathrm{~Hz} / \mathrm{s}$, thus replicating the same condition that occurred in South Australia before the blackout. For the sake of frequency resolution and estimation accuracy, the Discrete FT (DFT) is computed over a window of $1 \mathrm{~s}$, leading to a spectrum granularity of $1 \mathrm{~Hz}$. The figure shows that only $32 \%$ of energy is transmitted in the range between 48 and $52 \mathrm{~Hz}$, and that the rest is spread well beyond the fundamental tone. This result shows that an analysis limited to a narrow bandwidth surrounding the rated power system frequency would provide only a partial approximation of the grid representative signals. Indeed, any modeling approach relies on assumptions, whose verification is a necessary condition for obtaining accurate results. DFT-based 
approaches, intended for single narrow-band components, do not consider the entire Fourier series decomposition of the signal under test, therefore, they may provide an inaccurate representation when studying non-stationary signals.

The traditional approach towards power system analysis, on the one hand, has driven the development of network modeling techniques based on the concept of phasor. In this sense, the phasor extraction operation can be seen as a signal compression, driven by the necessity of providing a concise and exhaustive description of the information contained in the time-domain signals. On the other hand, the phasor concept has motivated also the implementation of system-awareness applications (e.g., PMUs and state estimators). However, the capability of accurately describing the power system behaviour is strongly related to the paradigm employed to formulate its governing physical laws. In this regard, power system operators are nowadays experiencing difficulties in interpreting phasor data estimated during large transients and are concerned about potentially wrong control actions relying on those estimates. The emerging trend is to build situational awareness systems that leverage on the raw time-domain data [17]. For instance, many PMU vendors are considering the idea of transferring the so-called point-on-wave data along with the PMU data stream. Those data could be collected at the data concentration point and then further analyzed by highly powerful computation tools. However this solution, besides being extremely bandwidth-demanding for the underlying telecommunication infrastructure, is simply postponing the problem of successfully compressing the information related to the considered power system event.

In this context, we are investigating mathematical transformations that enable us to go beyond the concept of phasor based on the FT: we envision a treatise in which the signal dynamics are preserved unaltered and thus can be suitably estimated, potentially leading to novel approaches for the operation and control of power networks. In this regard, the Hilbert Transform (HT) might represent a promising and effective solution. Given the acquired waveform, the HT produces a complex-valued signal, also known as analytic signal, whose spectrum consists only of real components [18]. Moreover, the HT may enable us to analytically formulate power system dynamics by means of dynamic phasors [19]-[22]. Also, analytic signals may be used to formulate circuit theory fundamental laws supporting frequency adaptive simulation of power system transients [23]-[27].

In this paper, we discuss the advantages of an HT-based representation of power system transients. First, we define equivalent expressions of signals representing typical power system dynamics and we derive their representation using the FT and the HT. Specifically, we consider (i) amplitude modulation, (ii) frequency ramp and (iii) amplitude step as representative transient operating conditions. The comparison of the obtained spectra enables us to highlight the limitation of FT-based analysis in non-stationary conditions and, conversely, the HT capability of accurately tracking the evolution of instantaneous power flows. Then we present a way to reconstruct the true instantaneous power using analytic signals. Finally, we validate these purely theoretical findings analyzing the results of dedicated time-domain simulations by means of the FT and HT. In particular, we use a simple 2-bus system to reproduce the three above defined transients as well as realworld datasets. We further use the IEEE 39-bus system to include a contingency analysis on a large scale power grid.

The paper is structured as follows. Section II provides the theoretical foundations. Section III describes the validation method. Section IV assesses the proposed approach performance in representative operative conditions. Section V concludes the paper discussing possible implications of the proposed HT-based analysis.

\section{RePRESEnTAtion OF POWER System TRANSIENTS USING THE HILBERT TRANSFORM}

This section aims at providing the theoretical basis of our investigation. First, Section II-A formulates typical power system transients using the FT and the HT, showing that, in principle, it is possible to build a solver that uses analytic signals to retrieve the instantaneous parameters associated to real power system signals. Such solver should integrate the Kirchhoff's circuit laws associated to the portion of the electrical grid under analysis as well as the expression of typical power system operating conditions. In the absence of an appropriate solver, whose formulation is out of the scope of this paper, Section II-B presents a way to reconstruct the true instantaneous power using analytic signals.

For the sake of nomenclature, we recall some definitions regarding the HT. Given a generic time-varying real-valued signal $x(t)$, its HT $\mathcal{H}(\cdot)$ is defined as [18]:

$$
\tilde{x}(t)=\mathcal{H}[x(t)]=\frac{1}{\pi} \int_{-\infty}^{+\infty} \frac{x(\tau)}{t-\tau} d \tau
$$

Such transform has the property of introducing a phase shift of $-\pi / 2$ at each positive frequency and $+\pi / 2$ at each negative frequency. The combination of the real signal $x(t)$ and its HT $\tilde{x}(t)$ form the so-called analytic signal $\hat{x}(t)$ :

$$
\hat{x}(t)=x(t)+j \cdot \mathcal{H}[x(t)]=x(t)+j \cdot \tilde{x}(t)
$$

\section{A. Application to Realistic Scenarios}

The HT enables us to analyze realistic signals typical of power system transients, where traditional FT-based techniques fail to give an appropriate phasor representation. For instance, let us start with the case of a generic power system signal whose amplitude is modulated by a cosine whose frequency is much slower than the fundamental one (i.e., the power system frequency). To fix ideas, this signal may represent a nodal voltage. This phenomenon typically appears during inter-area oscillations between large system regions. Formally, an amplitude modulation can be modeled as [28]:

$$
x(t)=A_{0}\left(1+k_{a} \cos \left(2 \pi f_{a} t\right)\right) \cdot \cos \left(2 \pi f_{0} t+\varphi_{0}\right)
$$

being $f_{0}, A_{0}$ and $\varphi_{0}$ the fundamental tone frequency, amplitude and initial phase, respectively, $f_{a}$ the modulation frequency $\left(f_{a} \ll f_{0}\right)$ and $k_{a}$ the modulation factor. 
In this case, the signal FT can be expressed as:

$$
\begin{array}{r}
\mathcal{F}[x(t)]=A_{0} / 2 \cdot\left[\delta\left(f-f_{0}\right) e^{j \varphi_{0}}+\delta\left(f+f_{0}\right) e^{-j \varphi_{0}}+\right. \\
\frac{k_{a}}{2} \cdot\left[\delta\left(f-\left(f_{0}+f_{a}\right)\right) e^{j \varphi_{0}}+\delta\left(f+\left(f_{0}+f_{a}\right)\right) e^{-j \varphi_{0}}+\right. \\
\left.\left.\delta\left(f-\left(f_{0}-f_{a}\right)\right) e^{j \varphi_{0}}+\delta\left(f+\left(f_{0}-f_{a}\right)\right) e^{-j \varphi_{0}}\right]\right]
\end{array}
$$

The spectrum is characterized by three pairs of bins in the positive and negative frequency domain: one at frequency $f_{0}$ and two centered around it at $f_{0} \pm f_{a}$. In such a scenario, an FTbased signal analyzer (tailored to investigate signals around the rated power system frequency) could fail to provide a correct spectrum interpretation because of the interference generated by the modulating tones. Conversely, the HT-based approach enables us to derive a single component analytic signal:

$$
\hat{x}(t)=A_{0}\left(1+k_{a} \cos \left(2 \pi f_{a} t\right)\right) \cdot e^{j\left(2 \pi f_{0} t+\varphi_{0}\right)}
$$

i.e., a phasor rotating at the fundamental frequency $f_{0}$, while its amplitude is pulsating at the modulation frequency $f_{a}$.

The second case under investigation consists in a power system whose frequency is decaying with a descending ramp trend, as typical of the stages anticipating a severe system collapse. A frequency ramp can be modeled as [28]:

$$
x(t)=A_{0} \cdot \cos \left(2 \pi f_{0} t+\varphi_{0}+R \pi t^{2}\right)
$$

being $R$ the ramp rate. The signal FT can be formulated as:

$\mathcal{F}[x(t)]=\frac{A}{2 \sqrt{2}} e^{j\left[\frac{\pi\left(f-f_{0}\right)^{2}}{R}-\frac{\pi}{4}+\varphi_{0}\right]}+\frac{A}{2 \sqrt{2}} e^{-j\left[\frac{\pi\left(f+f_{0}\right)^{2}}{R}-\frac{\pi}{4}+\varphi_{0}\right.}$

In this context, it should be noticed how difficult it is to distinguish the fundamental tone from the spurious contributions introduced by the time-varying frequency.

By contrast, the HT provides the following analytic signal:

$$
\hat{x}(t)=A_{0} \cdot e^{j\left(2 \pi f_{0} t+\varphi_{0}+R \pi t^{2}\right)}
$$

that can be regarded as a dynamic phasor, characterized by constant amplitude and rotating at the ramping frequency.

The final case of our investigation refers to a situation where FT-based methods provide largely discrepant results, i.e., a signal whose amplitude experiences a step, modeled as [28]:

$$
x(t)=A_{0}\left(1+k_{s} h(t)\right) \cdot \cos \left(2 \pi f_{0} t+\varphi_{0}\right)
$$

being $k_{s}$ the step factor and $h(t)$ the Heaviside function, that is null for $t<0$ and 1 for $t \geq 0$. The signal FT is:

$$
\begin{aligned}
\mathcal{F}[x(t)]= & A / 2\left(1+k_{s} / 2\right) \cdot\left[\delta\left(f-f_{0}\right) e^{j \varphi_{0}}+\delta\left(f+f_{0}\right) e^{-j \varphi_{0}}\right] \\
& +\frac{k_{s} A}{j 4 \pi\left(f-f_{0}\right)} e^{j \varphi_{0}}+\frac{k_{s} A}{j 4 \pi\left(f+f_{0}\right)} e^{-j \varphi_{0}}
\end{aligned}
$$

This formulation still maintains the information regarding the fundamental tone, but contains also two hyperbolic terms whose contributions are spread over the whole frequency spectrum. In such a scenario, an FT-based approach fails in providing an appropriate reconstruction of the signal under investigation due to the scattering of the spectrum bins that largely bias the information related to the fundamental tone.

The HT, instead, provides an analytic signal formulation:

$$
\hat{x}(t)=A_{0}\left(1+k_{s} h(t)\right) \cdot e^{j\left(2 \pi f_{0} t+\varphi_{0}\right)}
$$

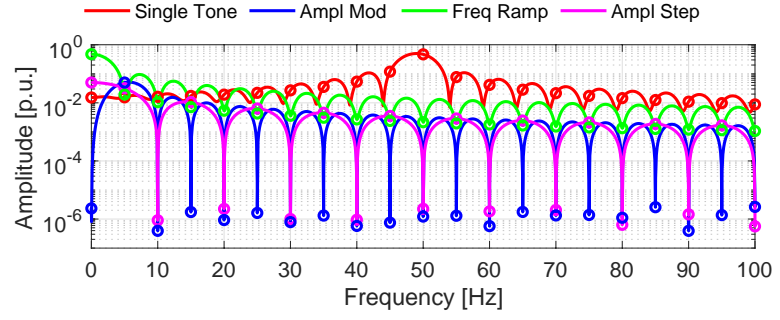

Fig. 2. DFT modules of a single tone cosine signal $y_{1}$ (red), of an amplitude modulation term $y_{2}$ (blue), of a frequency ramp term $y_{3}$ (green) and of an amplitude step $y_{4}$ (pink). The DFT is computed using an observation interval of $200 \mathrm{~ms}$ and a sampling frequency of $10 \mathrm{kHz}$

where the amplitude step information is entirely preserved.

Equations (5), (8) and (11) are derived assuming the following property of the Hilbert transform: the HT of the product of two signals with non-overlapping spectra is equal to the product of the low-frequency term by the HT of the high-frequency term [18]. In this regard, Fig. 2 shows the DFT modules of four signals, computed using an observation interval of $200 \mathrm{~ms}$ and a sampling frequency of $10 \mathrm{kHz}$. In particular, the red plot represents the spectrum of a single tone signal $y_{1}$ pulsating at $49 \mathrm{~Hz}$, with amplitude $A_{0}=1$ p.u. and phase $\varphi_{0}=0 \mathrm{rad}$. The blue plot represents the modulating term in (3), characterized by a typical modulation amplitude for power systems $k_{a}=0.1$ p.u., and modulation frequency $f_{a}=5 \mathrm{~Hz}\left(y_{2}(t)=k_{a} \cos \left(2 \pi f_{a} t\right)\right)$. The green plot refers to the frequency ramp in (6), with a ramping factor $R=-6.25 \mathrm{~Hz} / \mathrm{s}\left(y_{3}(t)=\cos \left(R \pi t^{2}\right)\right)$. The pink plot represents the amplitude step in (9), applied in the central point of the window, considering a typical step amplitude for power systems $k_{s}=0.1$ p.u. $\left(y_{4}(t)=k_{s} \cdot h(t)\right)$. As shown in the figure, in the frequency range $[45,55] \mathrm{Hz}$, the DFT bins corresponding to the fundamental tone $y_{1}$ are at least two orders of magnitude bigger than the DFT bins of the interfering tones $y_{2}, y_{3}$ and $y_{4}$. Therefore, even though it is not formally verified that the two signals are characterized by non-overlapping spectra, it is practically satisfied by typical power systems signals when the frequency resolution is in the order of few $\mathrm{Hz}$.

It is also worth pointing out that the current treatise refers to single tone signals. In the presence of interfering components, a suitable decomposition should be performed, like the one introduced by the Hilbert-Huang transform [29], [30].

\section{B. Reconstruction of the Electrical Power using the HT}

Let us consider two signals representing a generic voltage $v(t)$ and current $i(t)$ of a power system. The instantaneous power is computed as the product among the two waveforms:

$$
p(t)=v(t) \cdot i(t)
$$

By applying (2), the analytic signals associated to the voltage, current and instantaneous power can be expressed as:

$$
\begin{aligned}
\hat{v}(t) & =v(t)+j \cdot \mathcal{H}[v(t)]=v(t)+j \cdot \tilde{v}(t) \\
\hat{i}(t) & =i(t)+j \cdot \mathcal{H}[i(t)]=i(t)+j \cdot \tilde{i}(t) \\
\hat{p}(t) & =p(t)+j \cdot \mathcal{H}[p(t)]=p(t)+j \cdot \tilde{p}(t)
\end{aligned}
$$


Furthermore, if we compute the product between the analytic signals of voltage and current, we get:

$$
\begin{aligned}
\hat{p}^{\prime}(t) & =\hat{v}(t) \cdot \hat{i}(t)=[v(t)+j \cdot \tilde{v}(t)] \cdot[i(t)+j \cdot \tilde{i}(t)]= \\
& =v(t) i(t)-\tilde{v}(t) \tilde{i}(t)+j \cdot[\tilde{v}(t) i(t)+v(t) \tilde{i}(t)]
\end{aligned}
$$

In a similar way, the product between the analytic signals of voltage and current complex conjugate is:

$$
\begin{aligned}
\hat{p}^{\prime \prime}(t) & =\hat{v}(t) \cdot \operatorname{con} j(\hat{i}(t))=[v(t)+j \cdot \tilde{v}(t)] \cdot[i(t)-j \cdot \tilde{i}(t)]= \\
& =v(t) i(t)+\tilde{v}(t) \tilde{i}(t)+j \cdot[\tilde{v}(t) i(t)-v(t) \tilde{i}(t)]
\end{aligned}
$$

It is interesting to compute the sum of (14) and (15):

$$
\hat{p}^{\prime \prime \prime}(t)=\hat{p}^{\prime}(t)+\hat{p}^{\prime \prime}(t)=2 v(t) \cdot i(t)+2 j \cdot \tilde{v}(t) \cdot i(t)
$$

whose real part is proportional to the instantaneous power:

$$
\operatorname{real}\left(\hat{p}^{\prime \prime \prime}(t)\right)=2 p(t)
$$

At first, voltage and current signals can be modeled as sinusoids pulsating at the same frequency $f$ (similar results can be obtained also for more exhaustive formulations):

$$
v(t)=V \cos \left(2 \pi f t+\varphi_{v}\right), \quad i(t)=I \cos \left(2 \pi f t+\varphi_{i}\right)
$$

The instantaneous power is given by the waveforms' product: $p(t)=v(t) \cdot i(t)=\frac{1}{2} V I\left[\cos \left(\varphi_{v}-\varphi_{i}\right)+\cos \left(4 \pi f t+\varphi_{v}+\varphi_{i}\right)\right]$

As expected, the resulting instantaneous power pulsates at a frequency that is double the power system frequency. The voltage and current HTs are obtained by shifting the spectra by $\pm \pi / 2$, resulting in the following analytic signals:

$$
\begin{array}{r}
\hat{v}(t)=v(t)+j \cdot \tilde{v}(t)=V \cos \left(2 \pi f t+\varphi_{v}\right)+j \cdot V \sin \left(2 \pi f t+\varphi_{v}\right) \\
\hat{i}(t)=i(t)+j \cdot \tilde{i}(t)=I \cos \left(2 \pi f t+\varphi_{i}\right)+j \cdot I \sin \left(2 \pi f t+\varphi_{i}\right)
\end{array}
$$

If we compute the HT of the instantaneous power, we get:

$$
\tilde{p}(t)=\frac{1}{2} V I \sin \left(4 \pi f t+\varphi_{v}+\varphi_{i}\right)
$$

However, by comparing this result with (19), we notice that the information regarding the constant offset is lost. That is to say that the HT of the instantaneous power computed by simply using its definition is useless with respect to (19). Similarly, if we compute the analytic signal of $p(t)$, we get:

$$
\begin{array}{r}
\hat{p}(t)=p(t)+j \cdot \tilde{p}(t)=\frac{1}{2} V I\left[\cos \left(\varphi_{v}-\varphi_{i}\right)+\right. \\
\left.+\cos \left(4 \pi f t+\varphi_{v}+\varphi_{i}\right)+j \cdot \sin \left(4 \pi f t+\varphi_{v}+\varphi_{i}\right)\right]
\end{array}
$$

that, compared to (19), maintains the constant offset information, but introduces an imaginary complex term that does not have a clear physical meaning.

As in (14) and (15), $\hat{p}^{\prime}$ and $\hat{p}^{\prime \prime}$ can be computed as:

$$
\begin{array}{r}
\hat{p}^{\prime}(t)=V I \cdot\left[\cos \left(4 \pi f t+\varphi_{v}+\varphi_{i}\right)+j \cdot \sin \left(4 \pi f t+\varphi_{v}+\varphi_{i}\right)\right] \\
\hat{p}^{\prime \prime}(t)=V I \cdot\left[\cos \left(\varphi_{v}-\varphi_{i}\right)+j \cdot \sin \left(\varphi_{v}-\varphi_{i}\right)\right]
\end{array}
$$

Finally, as in (16), the sum of the obtained quantities is:

$$
\begin{aligned}
& \hat{p}^{\prime \prime \prime}(t)=V I \cdot\left[\cos \left(\varphi_{v}-\varphi_{i}\right)+\cos \left(4 \pi f t+\varphi_{v}+\varphi_{i}\right)\right]+ \\
& j \cdot V I \cdot\left[\sin \left(\varphi_{v}-\varphi_{i}\right)+\sin \left(4 \pi f t+\varphi_{v}+\varphi_{i}\right)\right]
\end{aligned}
$$

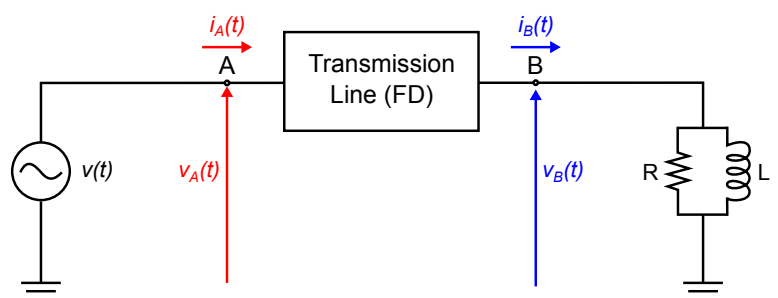

Fig. 3. Block diagram of the adopted EMTP-RV simulation model. The generator is characterized by nominal voltage of $380 \mathrm{kV}$, and is connected to a $100 \mathrm{MW}$ load through a $100 \mathrm{~km}$ frequency dependent (FD) overhead line.

whose real part corresponds to twice the instantaneous power in (19), exactly as in (17), demonstrating that $\hat{p}^{\prime \prime \prime}$ can be used to derive the instantaneous power of an electrical grid. The imaginary part, instead, does not have a specific physical meaning but is just a modeling artifact.

\section{VALidation Models AND ALgORITHM}

In this section, we provide the modeling and algorithmic details for the numerical validation of the proposed HT-based analysis. To this end, we use two models and two different simulation environments to generate datasets characterized by significant power system dynamics. First, we adopt a simple 2-bus model using the EMTP-RV simulation environment [31], [32], in order to replicate the waveforms described in Section II. Second, we use the IEEE 39-bus model [33], [34] implemented within the OPAL-RT environment [35], in order to emulate the operating conditions of large-scale networks. We further present an algorithm that enables us to compare the performance of FT- and HT-based approaches. It is worth pointing out that the FT is approximated by means of the DFT.

\section{A. Simple 2-bus Model}

We perform off-line simulations within the EMTP-RV simulation environment [31], [32]. Without loss of generality, in order to use a repeatable example, we refer to the simple model in Fig. 3, that consists of a time-varying 3-phase voltage generator powering a generic load through a transmission line, characterized by steady-state rated voltage of $380 \mathrm{kV}$ at $50 \mathrm{~Hz}$.

The voltage source has been modeled by means of a lookup-table providing point-on-wave data representing a generic dynamic voltage supplying the line. We consider a $100 \mathrm{~km}$ aluminum conductors steel reinforced overhead line modeled using the frequency dependent line model in [36]. The load, instead, is modeled by means of an R-L parallel equivalent, where $\mathrm{R}$ and $\mathrm{L}$ have been tuned in order to get a total load of $100 \mathrm{MW}$ and 0.9 power factor in case of a purely sinusoidal voltage at $50 \mathrm{~Hz}$.

\section{B. The IEEE 39-bus Model}

In order to test the proposed technique over large-scale power systems, we adopt the Opal-RT eMEGAsim PowerGrid Real Time Simulator (RTS) [35] to implement a detailed dynamic model of IEEE 39-bus power system, also known as 10machine New-England power system [33], represented in Fig. 4. This model represents a widely-employed benchmark for 


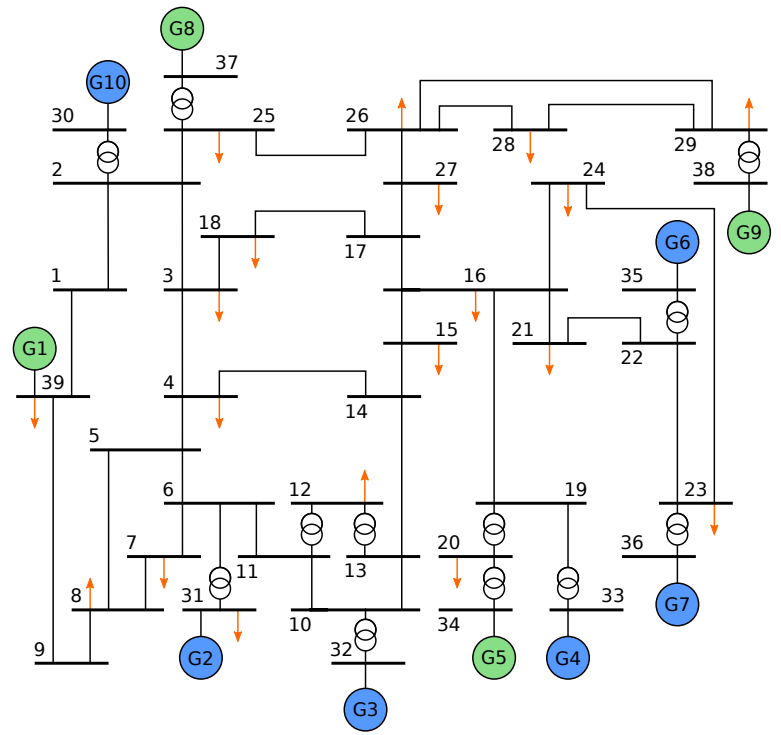

Fig. 4. Block diagram of the modified IEEE 39-bus power grid Opal-RT simulation model [34]. The system is characterized by nominal voltage of $345 \mathrm{kV}$. The model consist of 6 synchronous generators (blue circles), 4 wind farms (green circles) and 19 loads (orange arrows). The total installed capacity is $10 \mathrm{GW}$.

performance evaluation and comparison of several monitoring and control applications. In more detail, the simulated power system has a nominal voltage of $345 \mathrm{kV}$, and consists of 39 buses, 10 generators and 19 loads. In order to take into account the effects of distributed renewable generation, we modify the benchmark by replacing 4 conventional synchronous generators with wind farms [37]. Specifically, this reduced-inertia model includes wind farms instead of generators G1, G5, G8, and G9, for a total wind installed capacity of $4 \mathrm{GW}$. The total capacity for conventional synchronous generators is $6 \mathrm{GW}$, for an overall system installed capacity of $10 \mathrm{GW}$. Moreover, in order to emulate realistic load and generation patterns, we use wind and load profiles coming from real measurements. The network is modeled in Simulink and the simulations are run using the Opal-RT eMEGAsim RTS. More details about this model are provided in [34].

\section{The Validation Algorithm}

In the simulated scenario, we know the time-domain 3-phase waveforms of voltage and current in each node, and we postprocess them via DFT and HT, as described in Algorithm 1.

First, we define the true instantaneous power $p(t)$ as the product between voltage and current waveforms (line 1 in Alg. 1). We focus our investigations on the instantaneous power, to avoid the non-unique interpretation of active and reactive power (and the underlying hypothesis of steady-state phasor) in case of a broad signal spectrum [38]. The 3-phase power is obtained by summing the contribution of each phase $\{a, b, c\}$. In order to emulate more realistic operating conditions, we simulate the noise and measurement uncertainty by means of a purely additive and uncorrelated white Gaussian noise component (line 3 and 13). Specifically, we consider a signalto-noise ratio (SNR) equal to $80 \mathrm{~dB}$.
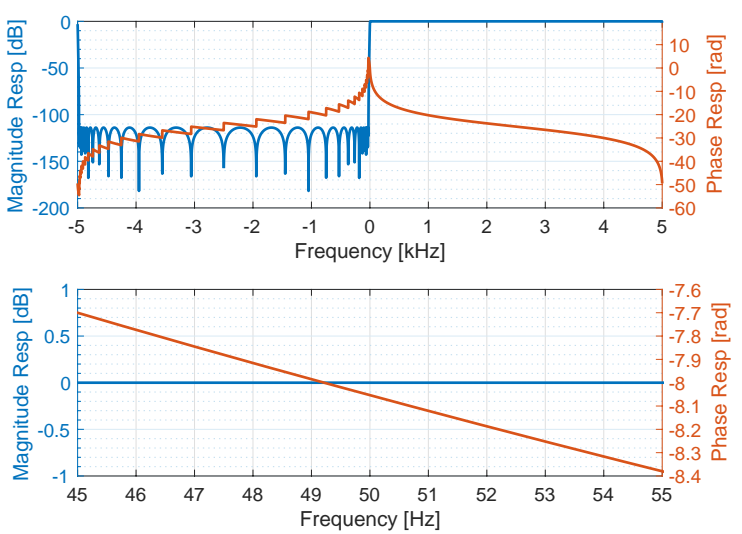

Fig. 5. Magnitude and phase response of the filter approximating the ideal HT over the whole frequency bandwidth (a) and over the power system operating conditions (b): filter order 31 and transition width $50 \mathrm{~Hz}$.

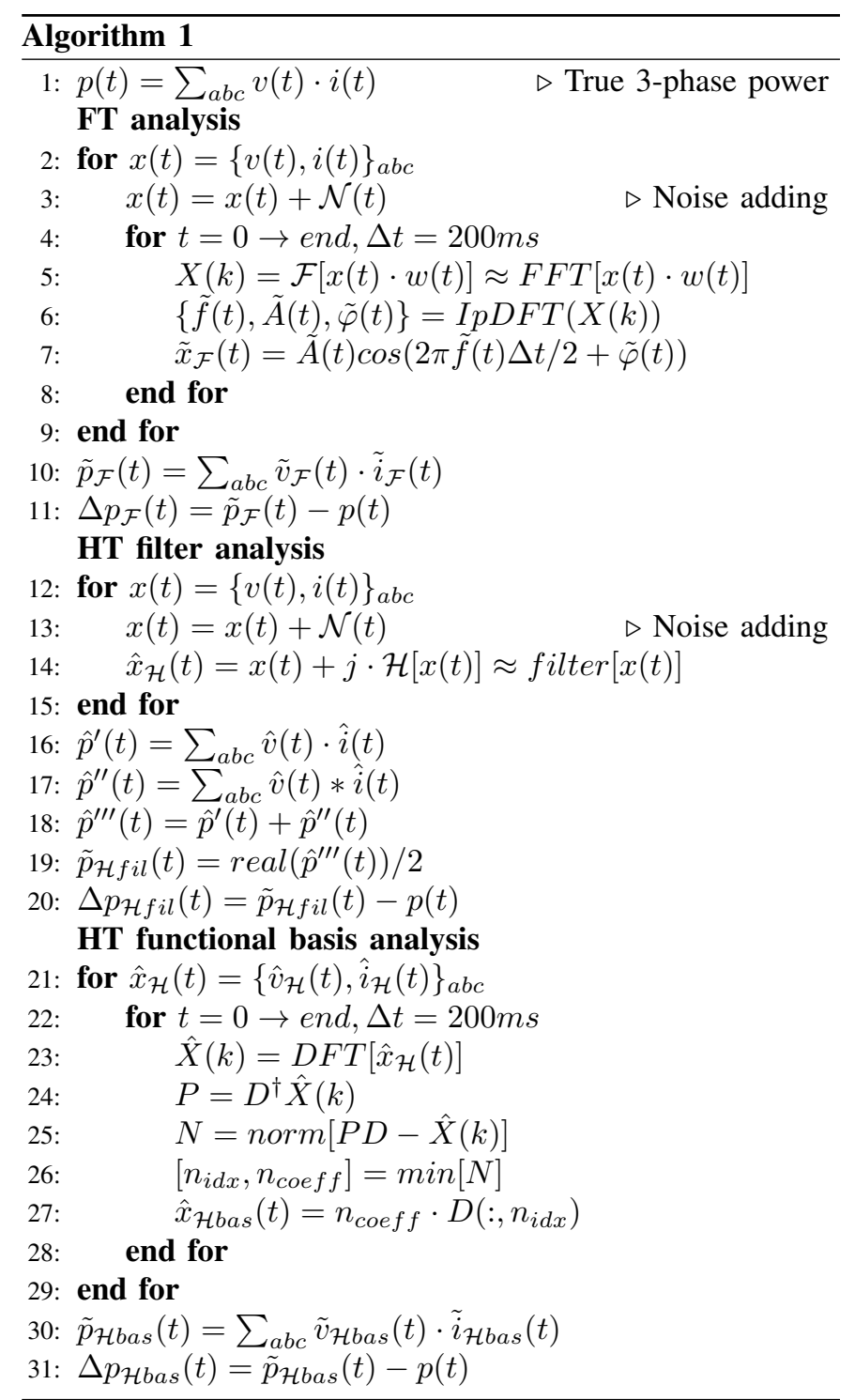


When the FT is adopted, we analyze the signals using a method inspired by the IEC Std. 61000-4-7 [39]: by means of sliding windows of $200 \mathrm{~ms}$, we shift the observation interval sample-by-sample (given the sampling frequency of $10 \mathrm{kHz}$, this corresponds to steps of $100 \mu \mathrm{s}$ ) (line 4). For each window, we approximate the FT by means of the DFT computed via a Fast FT (FFT) algorithm (line 5). The adopted window length (corresponding to 10 cycles at the nominal power system frequency) determine a $5 \mathrm{~Hz}$ granularity in the frequency domain. In order to reduce spectral leakage effects, we window the signal with the Hanning weighing function $w(t)$. In order to reduce the effects of spectrum granularity, for each observation interval we define the estimated frequency $\tilde{f}(t)$, amplitude $\tilde{A}(t)$ and phase $\tilde{\varphi}(t)$ by means of the Interpolated DFT (IpDFT) (line 6) [40], [41]. For the sake of conciseness, this analysis is not repeated for different interval lengths or windowing functions ${ }^{1}$. These estimates are used to approximate the time domain trend of the sinusoidal component in the considered observation interval. We construct the estimated signal $\tilde{x}_{\mathcal{F}}(t)$ by extracting the central point of each of the consecutive sliding windows (line 7). The 3-phase power is obtained by summing the contribution from each phase (line 10) resulting in power errors (line 11).

As regards the HT, we first compute the analytic signal of the noisy waveforms (line 14). We approximate the analytic signal by means of a filter using $10 \mathrm{kHz}$ sampling frequency, filter order set to 31 and transition width set to $50 \mathrm{~Hz}$, in order to comply with PMU-based applications [42]. As shown in Fig. 5, the magnitude response is nearly equal to zero (the ideal value) for the positive frequency domain, whereas it is lower than $-120 \mathrm{~dB}$ in the negative frequency range, achieving a high rejection of the long-range interference coming from the negative frequency components. As regards the phase response, an offset is introduced but the trend around the rated power system frequency is almost linear and can be characterized and compensated in post-processing.

Finally, we compute the quantities defined in equations (14)(16) (line 16-18). As in (17), the estimated power $\tilde{p}_{\mathcal{H}}(t)$ is computed as the real part of $\hat{p}^{\prime \prime \prime}(t)$ (line 19) and the corresponding power errors are consequently computed (line 20).

\section{Functional Basis Analysis}

An alternative solution for approximating the signal HT is provided by functional analysis [43]. The acquired signal $x(t)$ is projected over a pre-defined vector basis of analytic signals whose kernel is defined as:

$$
\left(1+g_{A}(t)\right) \cdot e^{j\left(2 \pi g_{f}(t) t+g_{\varphi}(t)\right)}
$$

where the functions $g_{A}, g_{f}$ and $g_{\varphi}$ account for the timevariations of amplitude, frequency and phase of the fundamental component, and might follow different trends like

\footnotetext{
${ }^{1}$ It is worth observing that as for any FT-based approach, the longer the observation interval, the smaller the spectrum granularity and thus the higher the accuracy of the resulting estimates. By contrast, a shorter interval length results in a faster response time in passing from one steady state to the other In this study we are mainly interested in the accuracy of the estimates, rather than on their responsiveness, therefore, we adopt a fairly long observation interval.
}

ramps, sinusoids or step changes. Differently from the filtering approach, the projection over a basis would allow to identify a mathematical model of the parameters' evolution that, de facto, would enable the compression of the time domain information into few coefficients of (27). In this regard, it is worth noticing that the employment of analytic signals enables us to define dictionary atoms that are not pure sinusoidal tones, but consist of modulated complex exponentials that better match the wideband spectrum of the dynamics under investigation. However, its performance depends on the number of possible realizations of (27) included in the basis: the larger the more accurate, but also the more computationally demanding and numerically illconditioned due to the high correlation between the vectors.

A possible solution via functional basis is provided in this Section, in order to demonstrate the prospective application of such an approach. In particular, a dictionary $D$ of vectors is constructed, that accounts for a limited set of disturbances that are likely to take place in the power system scenario. According to the IEC Std. 61000-4-7 [39], observation intervals of $200 \mathrm{~ms}$ are considered. More specifically, the frequency of the fundamental tone is limited to a finite bandwidth between 48 and $52 \mathrm{~Hz}$ with a step of $0.01 \mathrm{~Hz}$. Then, for each frequency, the following vectors are included in the basis:

- A steady-state sinusoid $\left(g_{A}=0, d g_{f} / d t=0, g_{\varphi}=0\right)$;

- A sinusoid characterized by an amplitude modulation $g_{A}(t)=1+k_{a} \cdot \cos \left(2 \pi f_{a} t\right)$, being $k_{a}=10 \%$ and $f_{a}$ $=[1,5] \mathrm{Hz}$ with steps of $0.01 \mathrm{~Hz}\left(d g_{f} / d t=0, g_{\varphi}=0\right)$;

- A sinusoid characterized by a frequency $\operatorname{ramp} g_{\varphi}(t)=$ $R \cdot \pi t^{2}$, being $R=[-6,6] \mathrm{Hz} / \mathrm{s}$ with steps of $0.01 \mathrm{~Hz} / \mathrm{s}$ $\left(g_{A}=0, d g_{f} / d t=0\right)$;

- A sinusoid characterized by an amplitude step $g_{A}=$ $k_{s} h_{s}(t-\tau)$, being $k_{s}=10 \%$ and $\tau=[0,200] \mathrm{ms}$ with steps of $5 \mathrm{~ms}\left(d g_{f} / d t=0, g_{\varphi}=0\right)$.

The dictionary contains the frequency domain representations of the so-defined analytic atoms.

The analytic signals computed in line 14 of Algorithm 1 are first divided into overlapping windows of $200 \mathrm{~ms}$, and then projected over the dictionary (line 24 in Alg. 1). Then, the residuals of the projection errors are computed (line 25) and the vector that minimizes the residuals is selected as solution (line 26). Finally, it is sufficient to multiply the selected vector for the corresponding projection coefficient to obtain the best approximated trend (line 27). The 3-phase power is obtained by summing the contribution from each phase (line 30) resulting in power errors (line 31).

\section{Results}

In this section, we describe the results that enable us to validate the proposed HT-based analysis. We analyze three different datasets. The first one is obtained using EMTP$\mathrm{RV}$ and is inspired by the signals formulated in Section II, representing approximations of actual power system operating conditions. The second dataset is still obtained using EMTPRV and refers to real-world events. Particularly, we replicate the waveforms taking place in Australia on September 2016 (see Fig. 1) and in Europe during an inter-area oscillation on December 2016 [11]. In that occasion, an unexpected 
opening of a line in the French transmission network caused a voltage phase angle difference in the continental Europe electricity system and decreased the general damping that triggered a permanent oscillation at $0.15 \mathrm{~Hz}$ [11]. The third set of waveforms is obtained simulating a large contingency within the IEEE 39-bus model using Opal-RT.

For each operating condition, the results are presented by means of two plots showing the instantaneous active power computed using the FT and the HT as in Alg. 1. The upper plot represents the power in phase $a$ and the true reference power, the bottom plot represents the 3-phase power error. In Section IV-A, the power errors are reported also for the functional basis analysis presented in Section III-D. The results are presented for one bus only, but similar considerations hold for the other buses of the considered network.

\section{A. Theoretical Operating Conditions}

By using Matlab environment, we numerically simulate a plausible situational awareness context, with a sampling frequency of $10 \mathrm{kHz}$. We synthesize voltage waveforms with a nominal voltage of $380 \mathrm{kV}$, for a total duration of 4 seconds for each test. These waveforms used to model the output of the voltage source are presented in Fig. 3. Then, as discussed in Section III, we analyze voltage and current signals as provided by EMTP-RV simulations. In more detail, we consider three cases with the signal characterized by:

a) Amplitude modulation, being $f_{a}=5 \mathrm{~Hz}$ the modulation frequency and $k_{a}=0.1$ the modulation factor in (3);

b) Negative frequency ramp in the range $50 \leq f_{0} \leq 46$, being $R=-6.25 \mathrm{~Hz} / \mathrm{s}$ the ramp rate in (6);

c) Amplitude step, being $k_{s}=0.1$ the step factor in (9).

Regarding the amplitude modulation case, Fig. 6 shows that the DFT does not enable us to correctly interpret a signal whose fundamental component is modulated. Indeed, the obtained signal spectrum is largely biased by the interference produced by the modulating term, leading to imprecise parameters estimation. Both voltage and current are inaccurately measured, leading to a maximum power error in the order of 50 MW. By contrast, it is evident that the HT, using the filter and the functional basis analysis, preserves the information needed to entirely reconstruct the analyzed signal: the maximum attained power errors are in the order of $10 \mathrm{~W}$.

Regarding the frequency ramp case, Fig. 7 shows that as the frequency deviates from its nominal value, the DFT provides erroneous results due to spectral leakage. The maximum obtained power error is in the order of $10 \mathrm{MW}$. Conversely, the HT is able to follow instantaneously the signal dynamics experienced during a frequency ramp, providing an almostperfect signal reconstruction. Also in this operating condition, the HT provides errors lower than $10 \mathrm{~W}$, both using the filter and the functional basis analysis.

Regarding the amplitude step case, the DFT spectrum does not contain the information needed to infer the signal parameters during such sudden waveform deformations. Fig. 8, shows that over the whole period during which the step is contained in the sliding window (i.e., $200 \mathrm{~ms}$ ), the DFT provides wrong results reaching $30 \mathrm{MW}$ error when the step appears in the
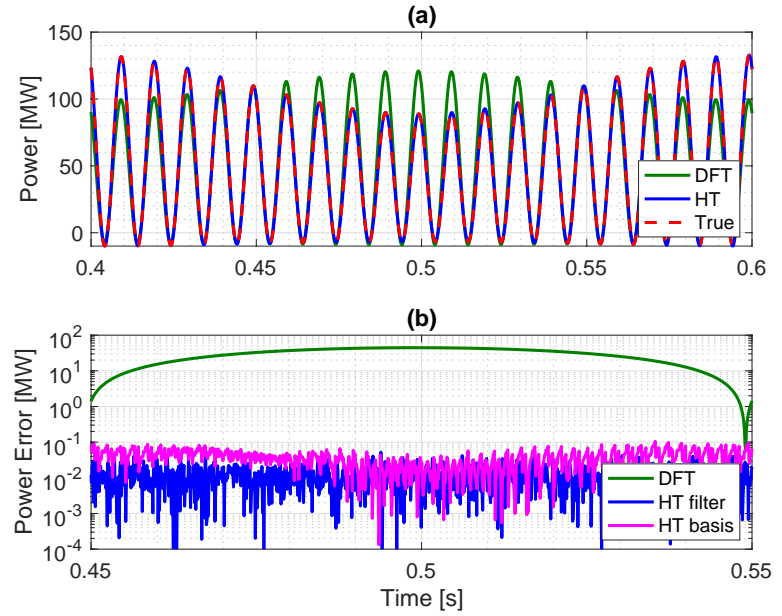

Fig. 6. Amplitude modulation: instantaneous single-phase active power (a) and three-phase power error (b) computed using the DFT (green) and the HT (blue) in case of a signal characterized by an amplitude modulation with modulating frequency of $5 \mathrm{~Hz}$. The red line represents the true power.

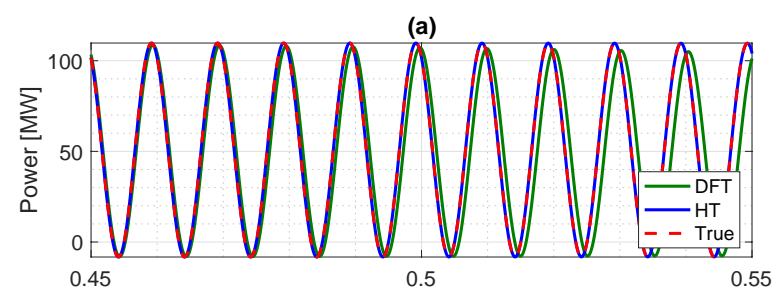

(b)

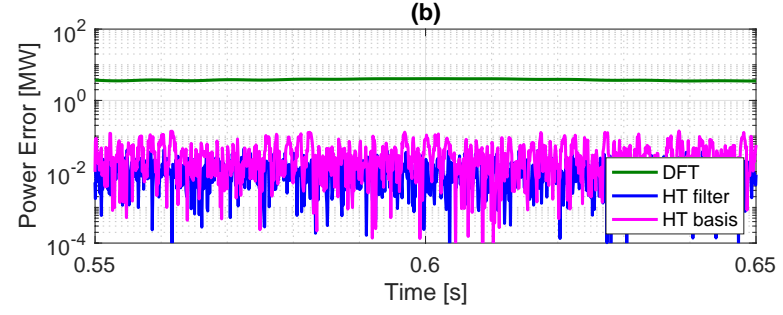

Fig. 7. Frequency Ramp: instantaneous single-phase active power (a) and three-phase power error (b) computed using the DFT (green) and the HT (blue) in case of a signal characterized by a negative frequency ramp of -6 $\mathrm{Hz} / \mathrm{s}$. The red line represents the true power.
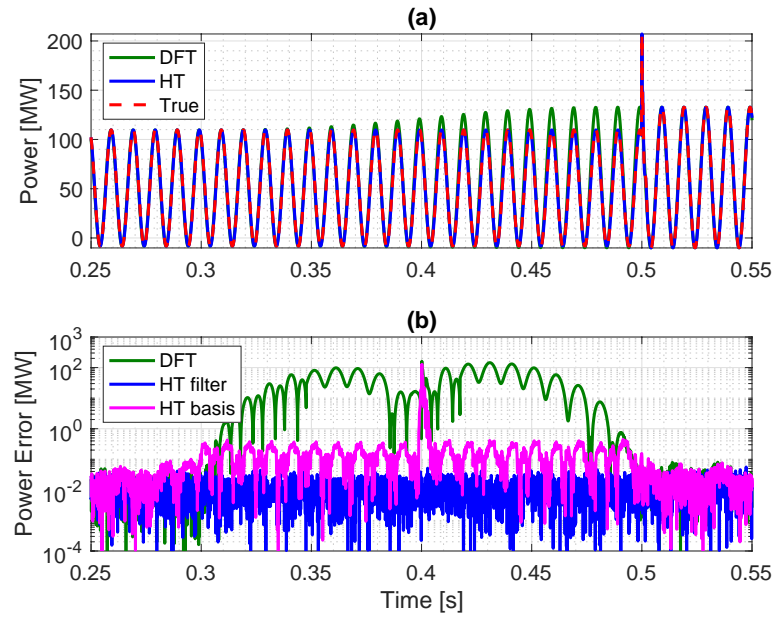

Fig. 8. Amplitude Step: instantaneous single-phase active power (a) and threephase power error (b) computed using the DFT (green) and the HT (blue) in case of a signal characterized by a $10 \%$ amplitude step. The red line represents the true power. 
center of the window and therefore the spectrum reconstruction is the most wanting. On the contrary, the HT enables us to follow the signal throughout the whole time interval, matching almost sample-by-sample the signal parameters. Again, the maximum attained power error is in the order of $10 \mathrm{~W}$. As regards the functional basis analysis, the figure shows that the proposed dictionary, that only accounts for ideal step changes, leads to errors in the same order of the DFT for few ms.

\section{B. Real-world Operating Conditions}

The second set of waveforms, refers to real-world events. Particularly, we replicate the waveforms taking place in Australia during the blackout on September 2016 and in Europe during the inter-area oscillations on December 2016 [10], [11]. Based on PMU estimates of fundamental frequency, amplitude and initial phase, at each reporting time the fundamental component trend is recovered in the time domain as sampled at $10 \mathrm{kHz}$, through the non-linear fitting approach in [28]. The method estimates the parameters of a series of samples by fitting them to a single-tone signal model. These datasets are used to replicate the voltage source in Fig. 3.

Regarding the Australian blackout, as illustrated in Fig 1 , the signal is characterized by two criticalities: first the amplitude step occurring at $1.6 \mathrm{~s}$, then the sudden frequency drop at $2.7 \mathrm{~s}$. As discussed so far, the two events are difficult to analyze adopting DFT-based tools because the signal spectrum is so spread that it is ambitious to infer the waveform parameters. Conversely, the HT proves to be potentially suitable for analyzing both situations. As a matter of fact, the results in Fig. 9 confirm the inappropriateness of the DFT to represent both the amplitude step and the frequency drop, exhibiting errors in the order of $100 \mathrm{MW}$. Besides, the HT is characterized by a maximum error lower than $10 \mathrm{~W}$.

Regarding the European inter-area oscillation, Fig. 10 confirms the improvement of adopting the HT in stead of the DFT, as the instantaneous power estimates are always characterized by a smaller error: maximum error lower than $10 \mathrm{~W}$ for the HT and $5 \mathrm{MW}$ for the DFT. The HT proves to be a suitable tool also for analyzing real-world waveforms.

\section{Large-scale Power System}

In order to evaluate the appropriateness of the proposed technique to model large-scale power grids, we carry out dedicated simulations of emergency scenarios using the IEEE 39-bus model in Opal-RT environment. In particular, we simulate the outage of generator G6, with a total tripped power of $800 \mathrm{MW}$, leading a load imbalance that determines a strong system dynamic. The generator is tripped at second 180 and the transient lasts for roughly 100 seconds.

Fig. 11 shows the power profiles recorded in bus 21 , but similar results hold for all the buses of the network. As it is shown in the figure, during the whole transient the DFT does not provide a truthful representation of the power system behavior, leading to errors in the order of $300 \mathrm{MW}$. Conversely, the HT provides an accurate transient tracking, with errors always lower than $800 \mathrm{~W}$. (a)

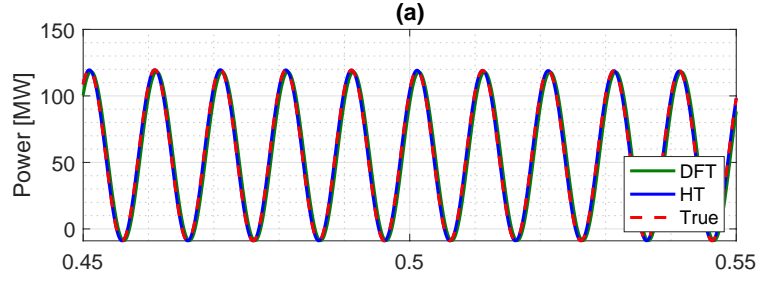

(b)

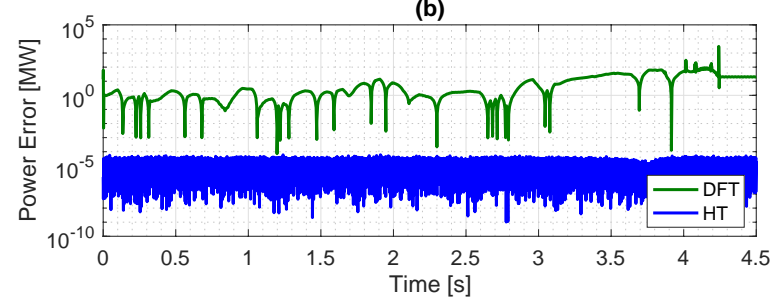

Fig. 9. Australian blackout: instantaneous single-phase active power (a) and three-phase power error (b) computed using the DFT (green) and the HT (blue) in case of replicating the waveforms that took place in Australia during the blackout on September 28, 2016. The red line represents the true power.

(a)

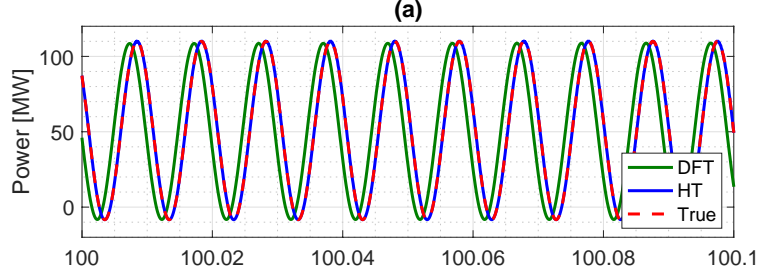

(b)

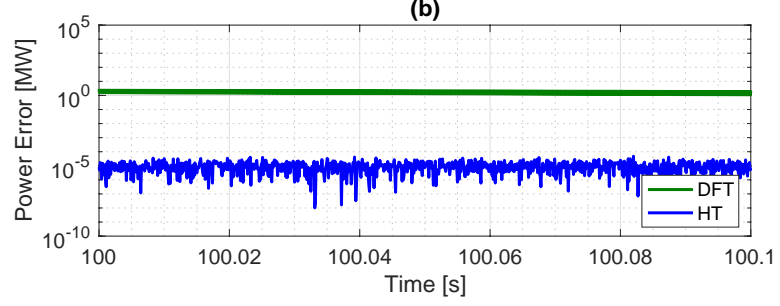

Fig. 10. European inter-area oscillation: instantaneous single-phase active power (a) and three-phase power error (b) computed using the DFT (green) and the HT (blue) in case of replicating the waveforms that took place in Switzerland during the inter-area oscillation on December 1, 2016. The red line represents the true power.
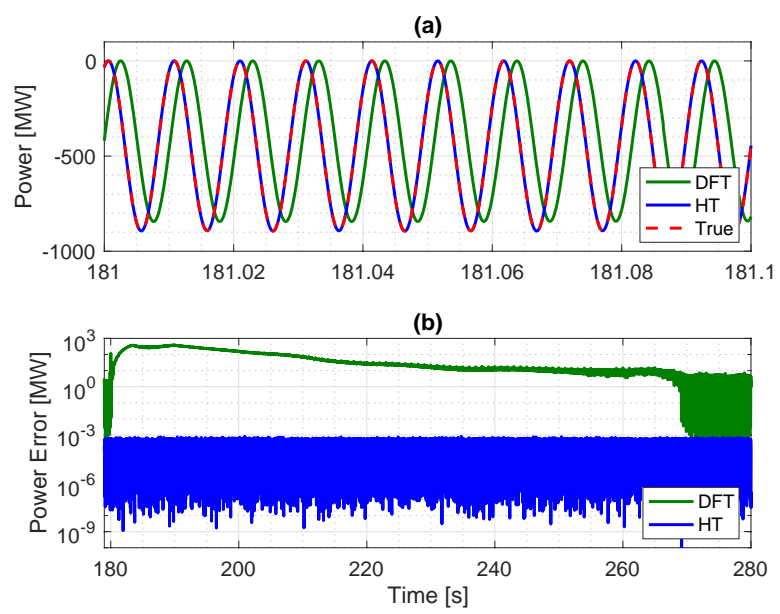

Fig. 11. IEEE 39-bus: instantaneous single-phase active power (a) and threephase power error (b) computed using the DFT (green) and the HT (blue) in case of tripping generator G6 at second 180. The power profiles refer to bus 21. The red line represents the true power. 


\section{Conclusions}

In this paper, we proposed an HT-based approach for studying broadband power system dynamics as an alternative to the traditional phasor-based representation. First, we compared the FT and HT of three large power system dynamics, and we discussed the limitations of FT-based analysis in non-stationary conditions, as well as the HT capability of tracking the evolution of the signals. Then, we carried out a numerical analysis where we developed two algorithms for the estimation of instantaneous power, relying on FT and HT representation, respectively. We characterized the accuracy to correctly compute the transmitted instantaneous power of the proposed transforms in both synthetic and real-world datasets. To this end, we carried out dedicated time-domain simulations.

Both theoretical and numerical results confirm that the HT is a suitable tool to be used in power system modeling and operation. Indeed, the HT provides superior results when computing the instantaneous power in all the considered operating conditions, leading to power errors up to 100 times smaller than in the case of using an FT-based representation. The treatise presented in this paper opens new scenarios for modern power systems modeling. On the one hand, HT-based situational awareness systems that rely on a broad spectrum could be deployed. For instance, we could think of PMUs capable of computing the analytic signal rather than the fundamental component synchrophasor. On the other hand, circuit theory fundamental laws could be formulated using analytic signals. Potentially, we could think of HT-based tools for power flow analysis.

\section{REFERENCES}

[1] F. Milano, F. Dörfler, G. Hug, D. J. Hill, and G. Verbič, "Foundations and challenges of low-inertia systems (invited paper)," in 2018 Power Systems Computation Conference (PSCC), June 2018, pp. 1-25.

[2] W. Winter, K. Elkington, G. Bareux, and J. Kostevc, "Pushing the limits: Europe's new grid: Innovative tools to combat transmission bottlenecks and reduced inertia," IEEE Power and Energy Magazine, Jan 2015.

[3] P. Tielens and D. V. Hertem, "The relevance of inertia in power systems," Renewable and Sustainable Energy Rev, vol. 55, pp. 999 - 1009, 2016.

[4] B. Kroposki et al., "Achieving a 100\% renewable grid: Operating electric power systems with extremely high levels of variable renewable energy," IEEE Power and Energy Magazine, vol. 15, no. 2, pp. 61-73, March 2017.

[5] D. Groß and F. Dörfler, "On the steady-state behavior of low-inertia power systems," IFAC-PapersOnLine, vol. 50, no. 1, pp. 10735 - 10741, 2017, 20th IFAC World Congress.

[6] F. R. S. Sevilla, P. Korba, K. Uhlen, E. Hillberg, G. Lindahl, and W. Sattinger, "Evaluation of the ENTSO-E initial dynamic model of continental Europe subject to parameter variations," in 2017 IEEE PES ISGT-Europe, April 2017, pp. 1-2.

[7] H. Pulgar-Painemal, Y. Wang, and H. Silva-Saravia, "On inertia distribution, inter-area oscillations and location of electronically-interfaced resources," IEEE Transactions on Power Systems, Jan 2018.

[8] N. Nguyen and J. Mitra, "Reliability of power system with high wind penetration under frequency stability constraint," IEEE Transactions on Power Systems, vol. 33, no. 1, pp. 985-994, Jan 2018.

[9] F. K. Tuffner, K. P. Schneider, J. Hansen, and M. A. Elizondo, "Modeling load dynamics to support resiliency-based operations in low-inertia microgrids," IEEE Transactions on Smart Grid, May 2019.

[10] AEMO, "Black system South Australia 28 September 2016," Australian Energy Market Operator, Tech. Rep., March 2017.

[11] ENTSO-E, "Analysis of CE inter-area oscillations of $1^{\text {st }}$ December 2016," European Network of Transmission System Operators for Electricity, Tech. Rep., 2017.

[12] A. Phadke and J. Thorp, Synchronized Phasor Measurements and Their Applications. Springer, Cham, 2017.
[13] J. D. L. Ree, V. Centeno, J. S. Thorp, and A. G. Phadke, "Synchronized phasor measurement applications in power systems," IEEE Transactions on Smart Grid, vol. 1, no. 1, pp. 20-27, June 2010.

[14] A. G. Phadke and B. Kasztenny, "Synchronized phasor and frequency measurement under transient conditions," IEEE Transactions on Power Delivery, vol. 24, no. 1, pp. 89-95, Jan 2009.

[15] D. Macii and D. Petri, "Harmonics estimation in transient conditions using static and dynamic frequency-domain techniques," in 2018 IEEE AMPS, Sep. 2018, pp. 1-6.

[16] M. Bertocco, G. Frigo, C. Narduzzi, C. Muscas, and P. A. Pegoraro, "Compressive sensing of a Taylor-Fourier multifrequency model for synchrophasor estimation," IEEE Transactions on Instrumentation and Measurement, vol. 64, no. 12, pp. 3274-3283, Dec. 2015.

[17] NASPI, "Data mining techniques and tools for synchrophasor data," NASPI Engineering Analysis Task Team, Tech. Rep., 2018.

[18] S. L. Hahn, Hilbert transforms in signal processing. Artech House, 1996.

[19] B. Boashash, "Estimating and interpreting the instantaneous frequency of a signal. I. fundamentals," Proceedings of the IEEE, vol. 80, no. 4 , pp. 520-538, April 1992.

[20] Z. Nowomiejski, "Generalized theory of electric power," Archiv für Elektrotechnik, vol. 63, no. 3, pp. 177-182, May 1981.

[21] P. C. See, V. C. Tai, M. Molinas, K. Uhlen, and O. B. Fosso, "Enhanced active power filter control for nonlinear non-stationary reactive power compensation," arXiv preprint arXiv:1206.4232, 2012.

[22] C. Qian and M. Kezunovic, "A power waveform classification method for adaptive synchrophasor estimation," IEEE Transactions on Instrumentation and Measurement, vol. 67, no. 7, pp. 1646-1658, July 2018.

[23] K. Strunz, R. Shintaku, and F. Gao, "Frequency-adaptive network modeling for integrative simulation of natural and envelope waveforms in power systems and circuits," IEEE Transactions on Circuits and Systems I: Regular Papers, vol. 53, no. 12, pp. 2788-2803, Dec 2006.

[24] P. Zhang, J. R. Marti, and H. W. Dommel, "Synchronous machine modeling based on shifted frequency analysis," IEEE Transactions on Power Systems, vol. 22, no. 3, pp. 1139-1147, Aug 2007.

[25] F. Gao and K. Strunz, "Multi-scale simulation of multi-machine power systems," International Journal of Electrical Power \& Energy Systems, vol. 31, no. 9, pp. 538-545, 2009.

[26] H. Ye and K. Strunz, "Multi-scale and frequency-dependent modeling of electric power transmission lines," IEEE Transactions on Power Delivery, vol. 33, no. 1, pp. 32-41, Feb 2018.

[27] M. Mirz, S. Vogel, G. Reinke, and A. Monti, "Dpsim—a dynamic phasor real-time simulator for power systems," SoftwareX, vol. 10, p. 100253 , 2019.

[28] G. Frigo, D. Colangelo, A. Derviškadić, M. Pignati, C. Narduzzi, and M. Paolone, "Definition of accurate reference synchrophasors for static and dynamic characterization of PMUs," IEEE Transactions on Instrumentation and Measurement, vol. 66, no. 9, Sept 2017.

[29] M. Sahani and P. K. Dash, "Automatic power quality events recognition based on Hilbert Huang transform and weighted bidirectional extreme learning machine," IEEE Transactions on Industrial Informatics, vol. 14, no. 9, pp. 3849-3858, Sep. 2018.

[30] I. Urbina-Salas, J. R. Razo-Hernandez, D. Granados-Lieberman, M. Valtierra-Rodriguez, and J. E. Torres-Fernandez, "Instantaneous power quality indices based on single-sideband modulation and wavelet packet-Hilbert transform," IEEE Transactions on Instrumentation and Measurement, vol. 66, no. 5, pp. 1021-1031, May 2017.

[31] J. Mahseredjian et al., "On a new approach for the simulation of transients in power systems," Electric Power Systems Research, vol. 77, no. 11, 2007, Selected Topics in Power System Transients - Part II.

[32] J. Mahseredjian, "Simulation des transitoires électromagnétiques dans les réseaux électriques," Édition Les Techniques de l'Ingénieur, 2008.

[33] T. Athay, R. Podmore, and S. Virmani, "A practical method for the direct analysis of transient stability," IEEE Transactions on Power Apparatus and Systems, vol. PAS-98, no. 2, pp. 573-584, March 1979.

[34] "IEEE-39-bus-power-system," https://github.com/DESL-EPFL/IEEE39-bus-power-system, accessed: 2019-06-01.

[35] "Opal-RT eMEGAsim PowerGrid real-time digital hardware in the loop simulator," https://www.opal-rt.com/, accessed: 2018-02-01.

[36] J. R. Marti, "Accurate modelling of frequency-dependent transmission lines in electromagnetic transient simulations," IEEE Transactions on Power Apparatus and Systems, vol. PAS-101, no. 1, pp. 147-157, Jan 1982.

[37] Y. Zuo, F. Sossan, M. Bozorg, and M. Paolone, "Dispatch and primary frequency control with electrochemical storage: a system-wise verification," in 2018 IEEE PES ISGT-Europe, Oct 2018. 
[38] A. Ferrero and G. Superti-Furga, "A new approach to the definition of power components in three-phase systems under nonsinusoidal conditions," IEEE Transactions on Instrumentation and Measurement, June 1991.

[39] “IEC Std 61000-4-7:2002(E)," Part 4-7: Testing and Measurement Techniques - General Guide on Harmonics and Interharmonics Measurements and Instrumentation, for Power Supply Systems and Equipment Connected Thereto, 2002.

[40] V. K. Jain, W. L. Collins, and D. C. Davis, "High-accuracy analog measurements via interpolated FFT," IEEE Transactions on Instrumentation and Measurement, vol. 28, no. 2, pp. 113-122, 1979.

[41] T. Grandke, "Interpolation algorithms for Discrete Fourier Transforms of weighted signals," IEEE Transactions on Instrumentation and Measurement, vol. 32, no. 2, pp. 350-355, 1983.

[42] G. Frigo, A. Derviškadić, and M. Paolone, "Reduced leakage synchrophasor estimation: Hilbert transform plus interpolated dft," IEEE Transactions on Instrumentation and Measurement, pp. 1-16, 2018.

[43] M. Vetterli, J. Kovačević, and V. K. Goyal, Foundations of Signal Processing. Cambridge University Press, Cambridge, 2014.

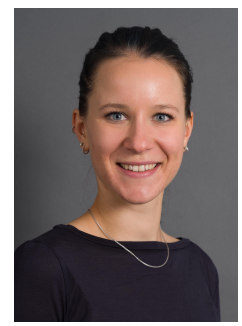

Asja Derviškadić (M'15) was born in Sarajevo, Bosnia and Herzegovina, in 1990. She received the Ph.D. degree in electrical engineering from the Swiss Federal Institute of Technology of Lausanne (EPFL), Lausanne, Switzerland in 2019 and the B.Sc. and M.Sc. degrees (Hons.) in electrical engineering from the University of Rome "La Sapienza," Rome, Italy, in 2012 and 2015, respectively. She is currently a Postdoctoral fellow at the Distributed Electrical Systems Laboratory of EPFL. Her research interests focus on synchronised sensing technologies for wide-area situational awareness of electrical grids operating in non-stationary conditions, including the development of enhanced Phasor Measurements Units (PMU).

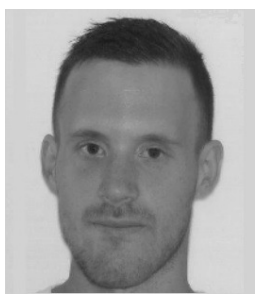

Guglielmo Frigo (M'18) was born in Padua, Italy, in 1986. He received the B.Sc. and M.Sc. degrees in biomedical engineering from the University of Padova, Padua, in 2008 and 2011, respectively, and the Ph.D. degree from the School of Information Engineering, University of Padova, in 2015, with a dissertation about compressive sensing (CS) theory applications to instrumentation and measurement scenario. He joined the Electronic Measurement Research Group, Department of Information Engineering, Padova, in 2011, and collaborated in the successful development of CS-based algorithms in the fields of spectral analysis, biomedical engineering, and smart-grid measurement. Since 2018 , he has been with the Distributed Electrical Laboratory, Swiss Federal Institute of Technology of Lausanne, Lausanne, Switzerland. He is currently Associate Editor of the IEEE Transactions on Instrumentation and Measurement, and member of the technical committee TC-39 Measurements in Power Systems as well as of the working group P1459 for Standard Definitions for the Measurement of Electric Power Quantities. His current research interests include the development of enhanced measurement devices for active distribution networks.

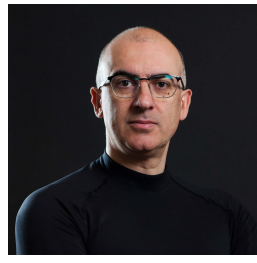

Mario Paolone (M'07, SM'10) received the M.Sc. (with Hons.) and Ph.D. degrees in electrical engineering from the University of Bologna, Italy, in 1998 and 2002, respectively. In 2005, he was appointed Assistant Professor in power systems with the University of Bologna, where he was with the power systems laboratory until 2011. In 2010, he received the Associate Professor eligibility from the Polytechnic of Milan, Italy. Since 2011, he joined the Swiss Federal Institute of Technology, Lausanne, Switzerland, where he is currently Full Professor,

Chair of the Distributed Electrical Systems Laboratory, Head of the Swiss Competence Center for Energy Research Future Swiss Electrical infrastructure and Chair of the EPFL Energy Centre Directorate. He has authored or coauthored over 300 papers published in mainstream journals and international conferences in the area of energy and power systems. His research interests focus on power systems with particular reference to real-time monitoring and operation aspects, power system protections, dynamics and transients. Dr. Paolone is the Editor-in-Chief of the Elsevier journal Sustainable Energy, Grids and Networks. 